n. 5, p. 28-37, 1993.

SCHNITMAN, A. Análise da fidedignidade da declaração da causa básica de morte por câncer em Salvador, Brasil. Rev. Saúde Pública, v. 24, n. 6, p. 490-6, 1990.

SOUZA, E.R. de. Violência velada e revelada: estudo epidemiológico da mortalidade por causas externas em Duque de Caxias, Rio de Janeiro. Cad. Saúde Pública, v. 9, n. 1, p. 48-64, 1993.

SZWARCWALD, C.L. \& CASTILHO, E.A. de. Mortalidade por causas externas no estado do Rio de Janeiro no período de 1976 a 1980. Cad. Saúde Pública, v. 2, n. 1, p. $19-41,1986$.

UNITED NATIONS. Levels and trends of mortality since 1950: a joint study by the United Nations and the World Health Organization. New York, 1982.

WORLD HEALTH STATISTICS ANNUAL, 1993. Genève: World Health Organization, 1994.

YUNES, J. Mortalidad por causas violentas en la región de las Americas. Bol. Oficina Sanit. Panam. *. 114, n. 4, p. 302-16, 1993.

\title{
AVALIAÇÃO "IN VITRO" DA ABRASÃO PRODUZIDA POR DENTIFRÍCIOS FLUORETADOS COMERCIAIS
}

\author{
SIMONIDES CONSAN| ${ }^{2}$ \\ MÁRIO FERNANDO DE GOES ${ }^{2}$ \\ MÁRIO ALEXANDRE COELHO SINHORETI \\ LOURENÇO CORRER SOBRINHO²
}

CONSANI, Simonides; GOES, Mário Fernando de; SINHORETI, Mário Alexandre Coelho; CORRER Sobrinho, Lourenço. Avaliação "in vitro" da abrasão produzida por dentifrícios fluoretados comerciais. Semina: Ci. Biol./Saúde, v. 16, n. 2, p. 308-312, jun. 1995.

RESUMO: A abrasão ocasionada por diversos dentifrícios durante a escovação foi avaliada em corpos de prova de acrilico (Plexiglas). O poder de abrasividade do agente parece depender mais da forma do que do tipo e tamanho das particulas quando pressão e velocidade de escovação são mantidas constantes. O dentifricio menos abrasivo foi o Prevent (Anakol) e o mais abrasivo o Signal G (Gessy-Lever).

Palavras-Chave: Escovação - dentifrícios - abrasão

\section{INTRODUÇÃO}

Promover higiene oral é remover placa dental e conseqüentemente prevenir o desenvolvimento de doenças no dente e nos seus tecidos de sustentação. Atualmente, a forma mais utilizada e conhecida para fazer higiene oral é a associação escova dentaldentifrícios (SVINNSETH et al.,1987).

Assim, os dentirícios na forma de cremes, pastas ou "géis" são utilizados para higienização bucal durante a escovação dos dentes. Embora tenham sido considerados cosméticos durante muito tempo, atualmente constituem-se na razão principal do surpreendente declínio de cárie dentária observado nos últimos dez anos na maioria dos países desenvolvidos (GLASS, 1982; HARGREAVES et al.,1983; JENKINS, 1985). Entretanto, isto só foi constatado nos países que fluoretaram seus dentifricios no início da década de 70 e uma das contraprovas é que no Japão a prevalência de cárie continua alta, devido à não utilização de flúor, segundo as afirmações de ROLLA (1990).
Porém, durante o processo de escovação ocorre uma certa abrasão (GRABENSTETTER et al., 1958; WRIGHT \& STEVENSON, 1967; STOOKEY \& MUHLER, 1968; BULL et al., 1968), provocada por agentes abrasivos contidos nos dentifrícios, ocasionando traumatismo nos tecidos duros dos dentes, moles da cavidade bucal e nas restaurações dentais. Esses danos ocorrem freqüentemente e estão associados a uma escovação regular com dentifrícios, com a predominância da retração gengival, seguida por erosões em forma de cunha na região da junção cemento-esmalte, causadas principalmente pelos agentes abrasivos e pela escova (SANGNES, 1976; SANGNES \& GJERMO, 1976).

No entanto, para que o dentifricio tenha propriedades teraupêuticas pela adição de flúor, em primeiro lugar deve ser satisfeita a compatibilidade química entre este e o sistema abrasivo. Abrasivos contendo cálcio reagem com ion flúor, insolubilizando-o e, portanto, diminuindo a concentração ativa para exercer efeito anti-cárie (CURY,1987; CURY, 1990; TEIXEIRA \& CURY, 1986).

1 - Realizaçăo do trabalho - Faculdade de Odontologia de Piracicaba / UNICAMP.

2 - Professores do Departamento de Odontologia Restauradora - Área de Materiais Dentários da Faculdade de Odontologia de Piracicabal UNICAMP - Av. Limeira, 901 - Piracicaba/SP - 13414018. 
O abrasivo mais utilizado no Brasil é o carbonato de cálcio, tendo em vista a fonte de matéria prima e o custo. Estima-se que as fábricas de pastas de dentes utilizem aproximadamente 30 toneladas de carbonato de cálcio por dia. Uma das alternativas para contomar o problema químico tem sido a adição de flúor não-iônico, por exemplo, na forma de monofluorfosfato de sódio (MFP). Porém, mesmo na presença de MFP uma certa porcentagem de flúor é inativado, atingindo, em função de uma série de condições, valores de no mínimo $20 \%$. Por outro lado, além do MFP ser importado, ele entra na formulação na proporção de $0,8 \%$, enquanto o NaF contribui com 0,2\% (CURY, 1990; STOOKEY, 1990). Outra alternativa seria a substituição do carbonato de cálcio por outro abrasivo compatível com flúor, por exemplo sílica; entretanto, enfrenta-se o problema não só do custo como da quantidade insuficiente no mercado, em função da demanda.

Além destes aspectos químicos considerados, outro fator importante é o grau de abrasividade dos dentifrícios, função das características físicas do abrasivo. Dessa forma, a abrasão na superfície dental é influenciada por várias propriedades do abrasivo, como composição química, estrutura cristalina, clivagem, friabilidade, solubilidade, concentração, dureza, tamanho e formato das partículas, assim como a compatibilidade com outros ingredientes da pasta (DAVIS, 1978; PUTT, 1979; REDMALM, 1986; BOER et al. 1985 ). Outras variáveis, como a marca, dureza das cerdas da escova dental e o baixo $\mathrm{pH}$ da solução pasta-saliva também influenciam no processo de abrasão da superfície dental, conforme afirmam HARTE \& MANLY (1975) e SVINNSETH et al. (1987).

Assim sendo, a proposta deste estudo foi avaliar "in vitro" a abrasividade de dentifrícios fluoretados comerciais sobre corpos de prova de acrílico.

\section{MATERIAL E MÉTODO}

Foram utilizados neste estudo 3 lotes diferentes de 21 marcas comerciais de dentifrícios fluoretados comumente encontrados no mercado brasileiro (Tabela 1).

Para a realização do ensaio de abrasão foram utilizadas escovas dentais Prevent 30, anti-placa, de cendas extra-macias de pontas arredondadas, dispostas em 3 fileiras de 10 tufos, contendo 40 cerdas em média em cada tufo, correspondendo a uma área de $192 \mathrm{~mm}$ (Anakol). A ponta ativa da escova era seccionada do cabo com disco de carboneto de silício (Viking) e fixada no dispositivo porta-escova da máquina de escovação com cola de secagem rápida (Super-Bonder, da Loctite), de modo a ficar com seu longo eixo perpendicular ao do corpo de prova.

A máquina de escovação utilizada foi de fabricação nacional (Equilabor), modificada do modelo indicado pela British Standard Institution - Especificação para cremes dentais, descrita no estudo de SLOP et al. (1983), com capacidade para 8 corpos de prova. O sistema propulsor da máquina permite um curso linear de varredura na faixa de $47 \mathrm{~mm}$, com velocidade controlada de 0 a 350 ciclos por minuto, registrada por um dispositivo de 4 dígitos.

Os corpos de prova medindo $47 \times 20 \times 2 \mathrm{~mm}$ foram confeccionados de placas de acrílico Plexiglas (Rohm \& Haas Co.), com dureza Vickers 20, e fixados com cera pegajosa no dispositivo porta-amostras, localizado no fundo do recipiente metálico de escovação do aparelho. Dada a característica comparativa do estudo, a escolha do material para a confecção do corpo de prova foi baseada nas afirmativas de WICTORIN (1972) e PANZERI et al. (1979), que o consideram um meio internacionalmente aceitável para investigar a abrasividade dos dentifrícios.

Em seguida, um volume de $4,6 \mathrm{ml}(6 \mathrm{~g})$ de dentifrício foi misturado a $6 \mathrm{ml}$ de água destilada $(6 \mathrm{~g})$ e vertido nesse recipiente metálico.

Os espécimens foram submetidos a movimentos lineares de escovação, com taxa de velocidade de 250 movimentos por minuto, totalizando 30.000 ciclos por amostra, num período de 2 horas. O percurso de escovação sobre o corpo de prova foi de $43 \mathrm{~mm}$, sob carga estática axial de $200 \mathrm{~g}$ colocada sobre o suporte do dispositivo porta-escova.

Os corpos de prova foram dispostos na máquina de escovação em 3 pares. Cada par foi submetido a uma amostra de dentifrício de lote diferente, assim como o corpo de prova controle. Após completado o ciclo de escovação, os corpos de prova eram removidos, lavados em água corrente, codificados de acordo com o dentifrício usado e armazenados em temperatura ambiente até o final dos ensaios. A cada ciclo, os corpos de prova, as escovas e os dentifrícios eram renovados.

Em seguida, os corpos de prova foram submetidos à leitura de rugosidade superficial produzida pela escovação, num aparelho PERTH-O-METER (Hannover, Alemanha). A leitura considerada foi a média aritmética entre picos e vales ( $\mathrm{Ra})$, com trecho percorrido pelo apalpador de $4,8 \mathrm{~mm}$ e trecho de medição de $4 \mathrm{~mm}$, com capacidade de medição $\mathrm{Ra}$ de +/-35 micrometros. Foram feitas 3 leituras em cada amostra (21 amostras do grupo controle e 126 amostras do experimental), totalizando 441 leituras. Os resultados foram submetidos à análise de variância e as médias comparadas pelo teste de Tukey (Tabela 2). Em seguida, os corpos de prova foram fotografados numa lupa estereoscópica Carl Zeiss (Alemanha).

\section{RESULTADOS}

A Tabela 2 mostra o teste de Tukey para as médias de rugosidade produzida pela abrasão dos dentifrícios durante a escovação. A menor média obtida foi apresentada pelo produto Prevent (Anakol) com valor $0,373333 \mu \mathrm{m}$ e a maior pelo dentifrício Signal G (GessyLever) com índice $6,073333 \mu \mathrm{m}$, quando comparadas com a média $0,019833 \mu \mathrm{m}$ obtida pelo padrão. Além do Prevent (Anakol), os produtos Fluotrat (BiolabSearle) e Kolynos anti-placa (Anakol) apresentaram 
médias de rugosidade sem diferença estatística significativa $(p<0,01)$ quando comparada com 0 padrão.

Os demais produtos apresentaram valores intermédiarios, todos com diferença estatística significativa $(p<0,01)$ com o padrão.

\section{DISCUSSÃO}

O dentifrício ideal seria aquele produto que promovesse a limpeza da superfície dental com o mínimo de abrasão, sem provocar efeitos irritantes à mucosa gengival. A maioria dos dentifrícios é formada por abrasivos, detergentes, umectantes, aglutinantes, misturas de aromatizantes, corantes, fluoretos e água.

Além de função terapêutica a que se propõem, a limpeza mecânica dos dentes é uma preocupação constante na formulação dos dentifrícios, pelo desgaste superficial que os agentes ocasionam na estrutura dental e nos materiais restauradores.

Os resultados obtidos em nosso trabalho (Tabela 2 e Figuras 1 a e b) demonstram o alto poder abrasivo da maioria dos 21 dentifricios, quando aplicados sobre corpos de acrílico (Plexiglas) numa varredura mecânica linear, com escovas dentais Prevent 30 (Anakol). Os dentifrícios estudados são formulados com abrasivos de alumina, carbonato de cálcio, fosfato de cálcio ou sílica. Segundo PANZERI et al. (1979), a abrasão provocada por esses agentes vai depender de outros fatores como, por exemplo, o tipo de escova dentária utilizada e as características dos hábitos de escovação, como freqüência, pressão exercida, tipo de movimento, etc. Nas condições de nosso trabalho, verificamos que 0 produto Signal $G$ (Gessy-Lever) contendo alumina como abrasivo foi o que provocou maior rugosidade superficial nos corpos de prova de resina (Plexiglas). O índice médio de rugosidade de 6,073333 $\mu \mathrm{m}$ alcançado por esse produto apresenta diferença estatística significativa $(p<0,01)$ quando comparado com o indice de 0,019833 $\mu \mathrm{m}$ obtido pela amostra padrão (escovação com água). Por outro lado, o menor índice médio de rugosidade superficial de $0,373333 \mu \mathrm{m}$ foi obtido pelo produto Prevent (Anakol), com sílica como abrasivo, sem mostrar diferença estatística significativa $(p<0,01)$, quando comparado com o padrão (Figuras $1 \mathrm{a} \mathrm{e} \mathrm{b).} \mathrm{Os} \mathrm{produtos}$ Kolynos Anti-placa (Anakol) e Fluotrat (Biolab-Searle), ambos formulados com sílica, apesar de apresentarem índices médios de rugosidade superficial maiores, não diferem significantemente $(p<0,01)$ com o apresentado pelo Prevent (Anakol).

Quando PANZERl et al. (1979) estudaram o poder abrasivo de 25 dentifricios aplicados com escova PróMulticendas sobre placas de Plexiglas (perda de peso), verificaram que o produto Kolynos Clorofila (Anakol) formulado com abrasivos de carbonato de cálcio apresentava um dos maiores índices de abrasividade. Em nosso trabalho, a rugosidade superficial média $(4,998333 \mu \mathrm{m})$ produzido por esse produto sobre corpos de prova de acrilico também foi bastante alto, não diferindo significativamente $(p<0,01)$ do dentifrício Signal
G (Gessy-Lever), com o mais alto índice de rugosidade superficial média $(6,073333 \mu \mathrm{m})$.

Além da interação com o meio utilizado como veículo (HARTE \& MANLY, 1975) e da relação técnicatempo de escovação (MOSS, 1972), a atuação do abrasivo depende do tipo, tamanho e forma de suas partículas (STOOKEY \& MUHLER, 1968). Assim sendo, segundo O'BRIEN (1989), a força aplicada sobre o abrasivo é de fundamental importância. Por essa razão é que, sob uma mesma força, partículas grandes e pequenas de formatos semelhantes produzem sulcos similares. Nas mesmas condições de força aplicada, as particulas afiadas produzem sulcos mais profundos do que as partículas arredondadas e uma mesma partícula produz sulcos mais largos e profundos com aumento da força aplicada. Também, a taxa de abrasão aumenta com o aumento da velocidade que o abrasivo percorre a superficie a ser abrasionada.

Portanto, em nosso trabalho a velocidade linear (250 ciclos por minutos) e a pressão da escovação (200g) foram constantes, assim como foi usado um único tipo de escova dental (Prevent 30), o que confere ao tipo e forma dos diversos agentes abrasivos a responsabilidade dos diferentes níveis de rugosidade superficial produzidos pelos dentifrícios (Figura 1).

Num estudo sobre a forma e distribuição de partículas abrasivas nos dentifrícios, PANZERI et al. (1979) verificaram que no produto Signal (Gessy-Lever), as partículas eram irregulares, com margens agudas e distribuição heterogênea, o que parece confirmar o alto indice médio de rugosidade apresentado em nosso trabalho pelos dentifrícios Signal G e Signal Plus. Já, dizem esses autores, as partículas usadas nos dentifrícios Colgate são arredondadas e a maioria apresenta forma irregular. Sua distribuição é homogênea, variando bastante o tamanho e algumas partículas apresentam bordas agudas. Os nossos resultados sobre rugosidade superficial mostram que os produtos da Colgate apresentaram valores intermédiarios (Colgate $\mathrm{MFP}+\mathrm{Ca}$, Colgate Gel, Colgate Gengi-Dent, Colgate AntiTártaro). Com respeito aos produtos Kolynos e Kolynos Gel, esses mesmos autores relatam que a forma das particulas abrasivas é bastante irregular, a distribuição altamente homogênea e os tamanhos os mais diversos, o que talvez tenha conferido aos produtos da Anakol os mais variados índices de rugosidade, verificados em nosso estudo, variando dos menores índices (Prevent, Kolynos Anti-Placa), intermediários (Fresh, Tandy, Kolynos Super Branco Pump) e até os maiores (Kolynos Clorofila, Flúor 2 Extra).

Outro dado interessante que notamos foi o fato da escova Prevent 30 (Anakol) produzir índice médio de rugosidade de $0,01933 \mu \mathrm{m}$ na amostra padrão (escovação com água), bastante próximo da rugosidade apresentada pelo corpo de prova de acrílico antes da escovação, isto é, indice de 0,01 simbolo $\mu \mathrm{m}$. Praticamente, esse tipo de escova não ocasionou abrasão no corpo de prova padrão, atuando apenas como agente mecânico na condução das partículas abrasivas sobre 
o corpo de prova durante o ciclo de escovação.

Isto significa que a associação escova dentalabrasivo deve ser prescrita de acordo com a freqüência clínica de escovação utilizada pelo cliente. Os dentifrícios menos abrasivos devem ser recomendados para aqueles pacientes que escovam os dentes várias vezes ao dia, porque o ato mecânico abrasivo repetitivo seria suficiente para desestruturar e remover a placa dental incipiente. Aos que menos escovam os dentes, os dentifrícios deveriam conter partículas mais abrasivas, que atuariam com maior eficiência na remoção da placa dental, que se acumula nos intervalos longos entre as escovações.

\section{CONCLUSÕES}

Os dentifrícios estudados apresentaram diferentes índices médios de abrasão quando associados a escovação linear de corpos de prova de acrílico (Plexiglas).

Não foi possível estabelecer uma relação entre diferentes tipos de abrasivo e taxa de abrasividade. Mantendo constante a pressão e velocidade de escovação, o poder de abrasividade do agente parece estar mais ligado à forma do que ao tipo e tamanho das partículas.

O dentifrício menos abrasivo foi o Prevent (Anakol) e o mais abrasivo o Signal $G$ (Gessy-Lever), sendo que a escova Prevent 30 (Anakol) não produziu abrasão superficial nos corpos de prova, que pudesse ser considerada relevante.

TABELA 1 - DENTIFRÍCIOS COMERCIAIS USADOS NO ESTUDO

\begin{tabular}{lll}
\hline \multicolumn{1}{c}{ Marca Comercial } & \multicolumn{1}{c}{ Tipo de Abrasivo } & Fabricante \\
\hline Xavier Anti-Cárie & Carbonato de Ca & Hepacholan \\
Sensodyne F & Fosfato de Ca & Billi Farm. \\
Fluotrat & Sílica & Biolab-Searle \\
Signal G & Alumina & Gessy-Lever \\
Signal Plus & Alumina & Gessy-Lever \\
Signal Anti-Tártaro & Sílica & Gessy-Lever \\
Close-up & Sílica & Gessy-Lever \\
Colgate Anti-Tártaro & Sílica e fosfato de Ca & Colgate \\
Colgate MFP & Carb. e fosfato de Ca & Colgate \\
Colgate MFP+Ca & Carb. e fosfato de Ca & Colgate \\
Colgate Gel & Silica & Colgate \\
Colgate Gengi-Dent & Sílica & Colgate \\
Kolynos Super & Carbonato de Ca & Anakol \\
Branco & & \\
Kolynos Clorofila & Carbonato de Ca & Anakol \\
Kolynos Star Gel & Sílica & Anakol \\
Kolynos Anti-Placa & Sílica & Anakol \\
Kolynos S.B.Pump & Silica & Anakol \\
Flúor 2 Extra & Carbonato de Ca & Anakol \\
Tandy & Sílica & Anakol \\
Fresh & Silica & Anakol \\
Prevent & Sílica & Anakol \\
\hline
\end{tabular}
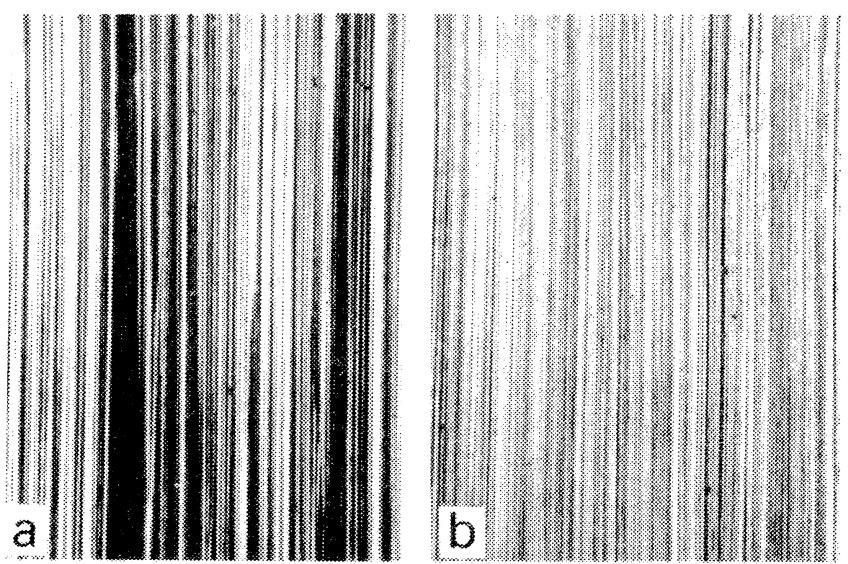

d

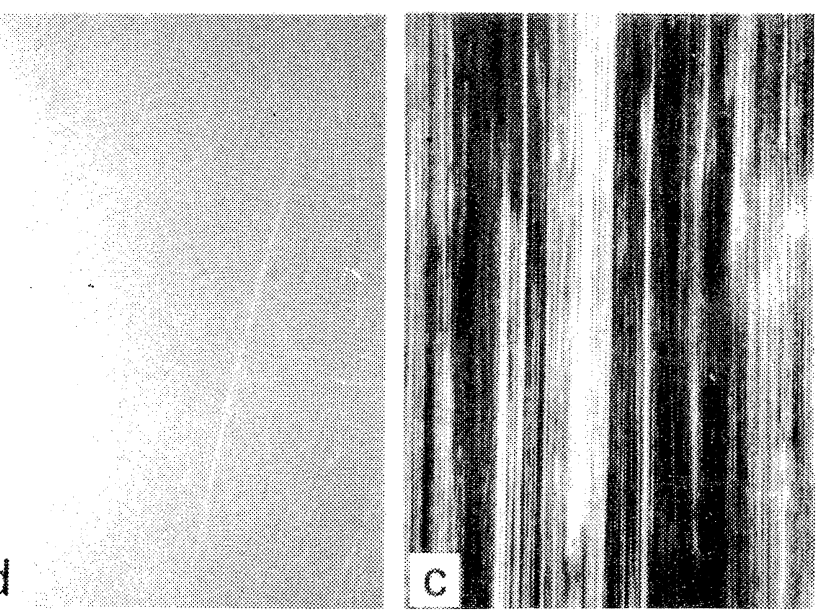

Figura 1 - Rugosidade superficial produzida pela escovação em corpos de prova de resina (Pexiglas) com os seguintes dentifrícios: a) Signal G (Gessy Lever): b) Prevent (Anakol); c) Kolynos antiplaca (Anakol). Em "d" observa-se a amostra padrão. (Aumento no negy ativo de 40 vezes

TABELA 2 - TESTE DE TUKEY PARA OS INDICES DE ABRASÃO $(\mu \mathrm{m})$ EM 6 REPETIÇÕES PARA CADA GRUPO EXPERIMENTAL E 21 REPETIÇÖES PARA O GRUPO CONTROLE

\begin{tabular}{|c|c|c|c|}
\hline$N^{0}$ & NOME & MEEDIAS & $1 \%$ \\
\hline 1 & Signal G & 6.073333 & A \\
\hline 2 & Signal Plus & 5.075000 & $A B$ \\
\hline 3 & Kolynos Clorofila & 4.998333 & $A B$ \\
\hline 4 & Flúor 2 Extra & 4.910000 & $A B$ \\
\hline 5 & Kolynos S. Branco & 4.766667 & $A B$ \\
\hline 6 & Close-up & 4.765000 & $A B$ \\
\hline 7 & Colgate Anti--Tártaro & 4.630000 & $A B C$ \\
\hline 8 & Colgate MFP & 4.621667 & $A B C$ \\
\hline 9 & Signal Anti-Tártaro & 4.601667 & $A B C$ \\
\hline 10 & Colgate Gengi-Dent & 4.026667 & $A B C D$ \\
\hline 11 & Anti-Cárie Xavier & 3.661667 & $B C D$ \\
\hline 12 & Kolynos Star Gel & 3.566666 & $B C D$ \\
\hline 13 & Colgate Gel & 3.240000 & $B C D$ \\
\hline 14 & Sensodyne $F$ & 3.058333 & $B C D$ \\
\hline 15 & Colgate MFP $+\mathrm{Ca}$ & 2.903333 & $B C D$ \\
\hline 16 & Kolynos S.B.Pump & 2.390000 & $\mathrm{CDE}$ \\
\hline 17 & Tandy & 2.296666 & CDEF \\
\hline 18 & Fresh & 2.141667 & DEF \\
\hline 19 & Fluotrat & 0.470000 & EF \\
\hline 20 & Kolynos Anti-Placa & 0.398333 & $E F$ \\
\hline 21 & Prevent & 0.373333 & $E F$ \\
\hline 22 & Padrão & 0.019833 & $F$ \\
\hline
\end{tabular}

Médias seguidas por letras distintas diferem entre si em nivel de $1 \%$ de significância.

D.M.S. $1 \%=2.33621$ 
CONSANI, Simonides; GOES, Mário Fernando de; SINHORETI, Mário Alexandre Coelho; CORRER Sobrinho, Lourenço. In vitro evaluation of abrasion produced by fluoridised commercial dentrifices. Semina: Ci. Biol./ Saúde, v. 16, n. 2, p. 308-312, Jun. 1995.

ABSTRACT: The abrasion produced by several dentrifice brands during brushing was evaluated on Plexiglas specimens. The agent abrasivity power seems to be more dependent on particle shape than on particle type and size, when brushing pressure and speed are maintained constant. The least abrasive dentifrice was Prevent (Anakol) and the most abrasive one was Signal G (Gessy-Lever).

KEY-WORDS: Toothbrushing - dentifrices - abrasion

\section{REFERÊNCIAS BIBLIOGRÁFICAS}

BOER, P.; DUINKERKE, A.S.H.; ARENDS J. Influence of toothpaste particle size and toothbrush stiffness on dentine abrasion in vitro. Caries Res., v. 19, p. 232-239, 1985.

BULL, W.H.; CALLENDER, R.M.; PUGH, B.R.; WOOD, G.D. The abrasion and cleaning propertes of dentifrices. $\mathrm{Br}$. Dent. J., v. 125, p. 331-337, 1968.

CURY, J.A. Estabilidade do flúor nos dentifrícios brasileiros. Rev.Gaucha Odont., v.46, n.4, p.36-40, 1987.

CURY, J.A. Evaluation of the fluoridated dentifrices sold in Brazil. J. Dent. Res., v.69, p.373, 1990. Sp Iss.

CURY, J.A. Flúor firmemente e fracamente ligado no esmalte e sua correlação com a composição dos dentifrícios fluoretados. In:VII REUNIĀO DA SOCIEDADE BRASILEIRA DE PESQUISA ODONTOLÓGICAS, 1990 Pirassununga.

DAVIS, W.B. The cleaning, polishing and abrasion of teeth by dental products. Cosmetic Science, v.1, p.39-81, 1978.

GLASS, R.L. First International conference on the declining prevalence of dental caries. J. Dent. Res., v. 61, p. 1304-1383, 1982. Sp Iss.

GRABENSTETTER, R.J.; R.W.; JACKSON, F.L.; RADIKE, A.W. The mesurament of the abrasion of human teeth by dentifrices abrasives: a test utilizing radioactive teeth. J. Dent. Res., v.37,p.1060-1068, 1958.

HARGREAVES, J.A.; THOMPSON, G.W.; WAGG, B.J. Changes in caries prevalence of Isle of Lewis Children between 1971 and 1981. Caries Res., v.17, p.554-9, 1983.

HARTE, D.B.; MANLY, R.S. Effect of toothbrush variables on wear of dentine produced by four abrasives. $J$. Dent. Res., v.54, p. 993-8, 1975.

JENKINS, G.W. Recent changes in dental caries. Br. Med. J., v. 291, p. 1297-8, 1985 .

MosS, A. Kliniske undersögelser over nogle tandpastaers og tandpulveres virkning over for plaque forekomst. Tandlaegebladet, v.75, p.197, 1971. In WICTORIN, L. Effect of toothbrushing on acrylic resin veneering material. Acta Odont.Scand., v. 30, n. 3, p. $383-395,1972$.

O'BRIEN, W.J. - Dental Materials: Properties and Selection. Berlin, Quintessence Publishing Co., Inc., 1989. $439 \mathrm{p}$.

PANZERI, H.; LARA, E.H.G.; SIESSERE, F.; MARCHETTI, R.M. Avaliação de dentifricios: parte 2: Forma e distribuição de partículas abrasivas. Odont. Mod., v.6, n. 2, p. 13-24, 1979.

PANZERI, H.; LARA, E.H.G.; SIESSERE, F.; MARCHETTI, R.M. Avaliação de dentifrícios. Parte 3 - Desgaste por escovação "in vitro". Odont. Mod., v.6, n.2, p.26-32, 1979.

PUTT, M.S. The development of a high polishing prophylaxis paste Thesis, Indiana University, 1979

REDMALM, G. Dentrifice abrasivity: the use of laser light for determination of the abrasives properties of different silicas. An in vitro study. Swed. Dent. J., v.10, p.243-250, 1986.

ROLLA, G. Some New Aspects of Preventive Dentistry. Faculdade de Odontologia de Piracicaba, 03 e 04 de Maio de 1990. (Curso).

SANGNES, G. Tramatization of teeth and gingiva related to habitual tooth cleaning procedures. J. Clin. Periodontal., v.3, p.94-103, 1976.

SANGNES, G.; GJERMO, P:: Prevalence of oral soft and hard tissue lesions related to mechanical toothcleasing procedures. Community Dent. Oral Epidemiol., v.4, p.77-83, 1976.

SLOP, D.; ROOIJ, J.F ; ARENDS, J. - Abrasion of enamel. I. An in vitro investigation. Caries Res., v.17, p.242-8, 1983.

STOOKEY, G.K. Critical evaluation of the composition and use of topical fluorides. J. Dent. Res., v.69, p.805-12, 1990. Sp Iss.

STOOKEY, G.K.; MUHLER, J.C. Laboratory studies concerning the enamel and dentin abrasion properties of common dentifrice polishing agents. J. Dent. Res., v. 47, p. 524-532, 1968.

SVINNSETH, P. N.; GJERDET, N.R.; LIE, T. Abrasivity of toothpastes: An in vitro study of toothpastes marketed in Norway. Acta Odont. Scand., v.45, p.195-202, 1987.

TEIXEIRA, R.N.; CURY, J.A. Reatividade dos dentifricios fluoretados comercializados no Brasil. Rev. Gaúcha Odont., v.34, n.5, p.381-3, 1986.

WICTORIN, L. Effect of toothbrushing on acrylic resin venneering material. II. Abrasive effect of selected dentifrices and toothbrushes. Acta Odont. Scand., v.30, n.3, p.383-395, 1972.

WRIGHT, K.H.R.; STEVENSON, J.I. The measurement and interpretation of dentifrices abrasiveness. $J$. Soc. Cosmet. Chem., v.18, p.387-411, 1967. 